\title{
Efecto de la fertilización orgánica foliar y al suelo con "Biol” sobre el rendimiento y sanidad de maíz (Zea mays), en el ciclo O-I en Sayula de Alemán, Veracruz, México
}

Effect of foliar and soil organic fertilization with "Biol" on performance and health of maize (Zea mays) in the O-I cycle Sayula German, Veracruz, México

Hernández Chontal Mario Alejandro ${ }^{1 凶}$, Ariadna Linares Gabriel ${ }^{1}$, Carlos Alberto Tinoco Alfaro $^{2}$ y Nereida Rodríguez Orozco ${ }^{1}$

${ }^{1}$ Universidad Veracruzana, Facultad de Ingeniería en Sistemas de Producción Agropecuaria. ${ }^{2}$ Instituto Nacional de Investigaciones Forestales, Agrícolas y Pecuarias.

${ }^{凶}$ Autor para correspondencia: maher_chontal@hotmail.com

Recibido: $13 / 01 / 2014$
Aceptado: 14/07/2014

\section{RESUMEN}

En la actualidad la producción de maíz decrece debido a la degradación de los suelos con niveles bajos de fertilidad, aunado a altos costos de producción y que además las técnicas de producción y las condiciones ambientales favorecen la presencia de plagas y enfermedades, que impacta negativamente sobre la producción. El biol es un biofertilizante que contiene microorganismos que al estar disponibles en las plantas le ayudan a su nutrición y protección (Vessey, 2003), además de ser un complemento a la fertilización tradicional, debido a la actividad microbiana de la que provienen, puede impactar positivamente sobre la sanidad de mazorcas y como tal obtener mejores rendimientos. Por lo anterior se planteó un diseño experimental con el objetivo de evaluar el rendimiento, sanidad y análisis económico de cada una de las combinaciones de biol. La investigación se llevó a cabo en Sayula de Alemán, Veracruz, se utilizó un arreglo de bloques al azar con parcelas divididas evaluándose en parcelas grandes la aplicación de biol al suelo y en parcelas chicas fertilizantes foliares a base de biol. Se obtuvo como resultados que las aplicaciones de biol + harina de ave + melaza mejoraron la sanidad de mazorcas y generó el mayor rendimiento de grano de $2.79 \mathrm{t} \mathrm{ha}^{-1}$, además que los menores rendimientos estuvieron asociados con los mayores porcentajes de daño a mazorcas ocasionados por el hongo Fusarium moniliforme.

Palabras clave: Biol, Fusarium moniliforme, Sanidad. 


\section{ABSTRACT}

Today corn production decreases due to degradation with low levels of fertility, coupled with high production costs and also production techniques and environmental conditions favor the presence of pests and diseases, which negatively impacts production. The biol is a biofertilizer containing microorganisms to be available in plants help your nutrition and protection (Vessey, 2003), and is a complement to traditional fertilization due to microbial activity coming, may impact positively on the health of ears and as such get better returns. Therefore experimental design was proposed in order to evaluate the performance, health and economic analysis of each of the combinations biol. The research was conducted in Alemán Sayula, Veracruz, we used a randomized block arrangement with split plot evaluated on large plots of biological application to soil and foliar fertilizers subplots based biol. Such analysis indicated that applications boil + poultry meal + molasses, improved health of ears and resulted in the highest grain yield of $2.79 \mathrm{t} \mathrm{ha}^{-1}$, in addition to the lower yields were associated with higher rates of damage to ears caused by Fusarium moniliforme.

Keywords: Biol, Fusarium moniliforme, health.

\section{INTRODUCCIÓN}

En el municipio de Sayula de Alemán, el maíz es cultivado por pequeños agricultores que destinan el $70 \%$ de la producción para autoconsumo y el resto para su comercialización, por lo anterior, esta especie es la de mayor importancia económica y social, lo cual se corrobora con las estadísticas del SIAP en el 2011, en las que se reporta una superficie aproximada de 2,000 hectáreas, con rendimientos bajos de 2 a $2.5 \mathrm{t} \mathrm{ha}^{-1}$ y con problemas de calidad del grano por las afectaciones que sufre por el ataque de hongos, que le provocan pudriciones que pueden llegar a disminuir el rendimiento hasta en un $50 \%$ (Tinoco et al. 2002). La baja productividad y calidad del maíz, se explica por la degradación de los suelos y las bajas dosis de fertilización (Tinoco et al. 2008). El objetivo de la investigación fue evaluar el efecto del biol con aplicación foliar y al suelo sobre el rendimiento y sanidad de mazorcas, así como realizar un análisis económico de cada uno de los tratamientos.

\section{MATERIALES Y MÉTODOS}

La investigación se realizó en la localidad de Almagres perteneciente a Sayula de Alemán, Veracruz, México, durante el ciclo otoño-invierno, utilizando semilla de maíz comercial Piooner 30F96, teniendo una densidad de población de 62,500 plantas por ha${ }^{1}$, con dos plantas por mata. Se manejó un experimento bajo un diseño de bloques al azar, con arreglo de parcelas divididas y cuatro repeticiones, evaluándose en parcelas grandes la aplicación de biol al suelo y en parcelas chicas fertilizantes foliares a base de biol (Cuadro 1), utilizando una dosis de dos litros por hectárea de las combinaciones de biol foliar aplicando 10 mililitros por cada 36 metros lineales, realizando tres aplicaciones a partir de los 15 días después de la emergencia, posteriormente a los 30 y 45 días; además se hizo una sola 
aplicación de biozyme al segundo nudo de la planta de maíz con una dosis de $250 \mathrm{ml} / \mathrm{h}^{-1}$. Para la aplicación de biol al suelo se utilizó una dosis de $150 \mathrm{l} / \mathrm{h}^{-1}$ de la combinación de biol $(90 \%)+$ harina de ave $(10 \%)$ realizando tres aplicaciones a partir de los 15 días después de la emergencia, posteriormente a los 30 y 45 días; se aplicaron 5 mililitros de biol por mata. La superficie total del experimento fue de 863 $\mathrm{m}^{2}$ con unidades experimentales de $14.4 \mathrm{~m}^{2}$. Las variables que se midieron fueron sanidad de mazorca, rendimiento de grano y la comparación de costos de los mejores tratamientos con la fertilización tradicional.

Para medir el rendimiento de grano se cosechó uno de los cuatro surcos de cada unidad experimental, se desgranó para obtener el peso y después calcular el rendimiento total por $\mathrm{ha}^{-1}$. Para medir la sanidad de mazorcas se tomaron cinco mazorcas de cada surco cosechado y se calificaron con una escala, tomando en cuenta el porcentaje de daño (Cuadro 2), para después realizar los análisis estadísticos. Para realizar el análisis económico de cada uno de los tratamientos se consideraron las variables rentabilidad, ganancia, costo variable, producción total, costo total e ingresos por venta. Los análisis estadísticos de las variables de estudio se realizaron a través del

Paquete de diseños experimentales F.A.U.A.N.L. Versión 2.5 (Olivares, 1994).

Cuadro 1: Tratamientos de las combinaciones de biol.

\begin{tabular}{l|l}
1 & Biol (70\%), Harina de ave (10\%), Suero (10\%) y melaza $(10 \%)$ \\
\hline 2 & Biol (70\%), Harina de ave (10\%) y Suero (10\%) \\
3 & Biol (70\%), Harina de ave (10\%) y melaza $(10 \%)$ \\
4 & Biol (70\%), Suero (10\%) y melaza $(10 \%)$ \\
5 & Biozyme \\
\hline &
\end{tabular}

Cuadro 2: Escala de calificación de sanidad de mazorcas.

\begin{tabular}{lll}
\multicolumn{1}{c}{ Escala } & \% de daño \\
\hline \hline 1 & Muy mala & $>20 \%$ \\
2 & Mala & $10-20 \%$ \\
3 & Buena & $6-10 \%$ \\
4 & Muy buena & $2-6 \%$ \\
5 & Excelente0 $-2 \%$ \\
\hline
\end{tabular}

\section{RESULTADOS Y DISCUSIÓN}

\section{Peso de grano}

Para la variable peso de grano, de acuerdo al análisis de varianza realizado se encontró diferencia significativa $(\mathrm{P}=0.038)$ y con base a la prueba de medias de Tukey los mejores resultados fueron para las combinaciones de biol + harina de ave + melaza, biol + suero + melaza y biozyme, con valores de 2.3 a $2.7 \mathrm{t} / \mathrm{ha}^{-1}$. El cual supera al

Revista Científica Biológico Agropecuaria Tuxpan 2 (1) 
promedio de $2.037 \mathrm{t} \mathrm{ha}^{-1}$ reportado en el estado para el ciclo otoño-invierno (SAGARPA,

2008).

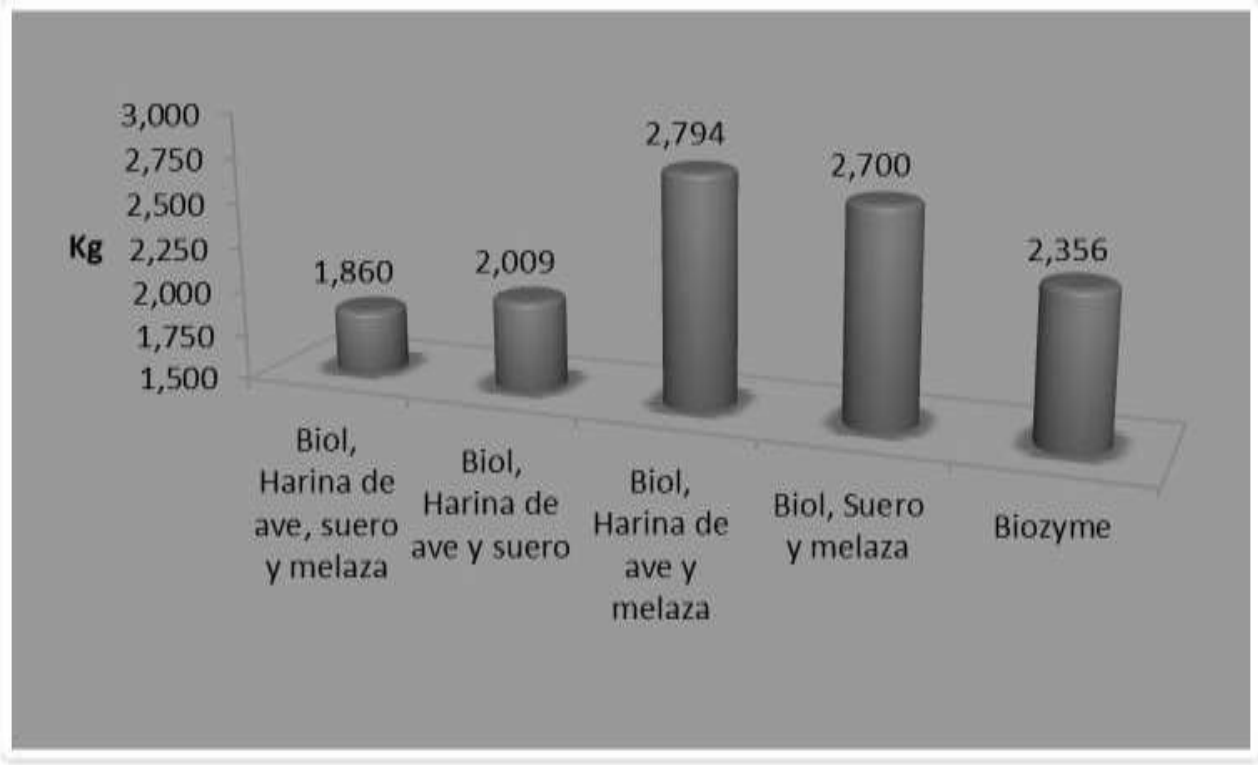

Figura 1. Medias de la variable peso de grano.

Los estudios coinciden con Bejarano y Méndez (2004), donde mencionan que el fitoestimulante biol al 5\% contribuyó a incrementar la producción de fríjol más que la fertilización con húmus de lombriz y biol al 10\%. Al igual Guanopatín, (2012) realizó un estudio de aplicación de biol y encontró un incremento en el rendimiento, en el cultivo de alfalfa (Medicago sativa).

\section{Sanidad de mazorcas}

El análisis de varianza mostró diferencia significativa $(\mathrm{P}=0.018)$ para la aplicación de combinaciones de biol, donde el mejor resultado fue el que tuvo la aplicación con la combinación de biol + harina de ave + melaza, que mostró según la escala el valor tres= buena sanidad de mazorcas. La variable sanidad de mazorcas mostró interacción para los niveles de los factores, observándose diferencia altamente significativa $(\mathrm{P}=0.006)$, y de acuerdo con la prueba de medias de Tukey se observó que el mejor tratamiento para la parcela con aplicación de biol al suelo fue la combinación de biol + harina de ave + melaza, mostrando según la escala el valor cuatro= muy buena sanidad de mazorcas; y para la parcela sin aplicación de biol al suelo, fueron los tratamientos con las combinaciones de biol + suero + melaza, biol + harina de ave + melaza, biol + harina de ave + suero y biozyme, según la escala el valor $3=$ buena sanidad de mazorcas. 


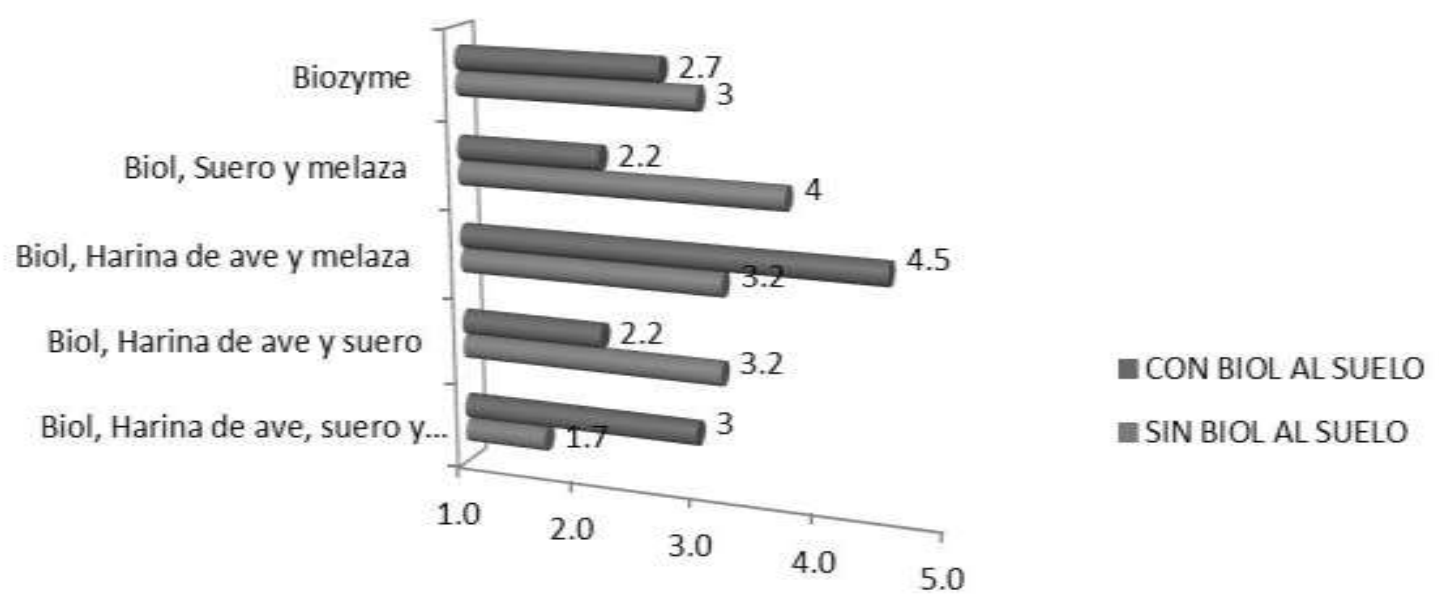

Escala Sanidad de mazorcas

Figura 2. Resultados de Sanidad de mazorcas mediante la escala del 1 al 5 (Cuadro 2).

Los resultados encontrados difieren de

los encontrados por Hernández y Esquivel en 2004, quienes no encontraron diferencias estadísticas al evaluar la variable sanidad de mazorcas en germoplasma de maíz de valles altos de México, la sanidad de mazorcas la evaluaron mediante una escala con valores de 1 al 9 , en donde $1=$ mazorcas completamente dañadas y $9=$ mazorcas sin daño, que incluyo daños por plagas y enfermedades. Sierra et. al., en el 2004, indicaron que entre mayor sea el daño por pudriciones, el rendimiento de grano es menor, ya que encontraron diferencia altamente significativa entre rendimiento de grano y pudrición de mazorca en 64 hibridas evaluados en el sureste de México.

\section{Análisis económico}

Se calcularon costos de producción de cada uno de los tratamientos de fertilizantes foliares, teniendo el mayor costo la aplicación del biofertilizante comercial con una diferencia de $\$ 47.6$ en relación con la aplicación del

biofertilizante. El mejor tratamiento correspondiente a la aplicación foliar fue biol + harina de ave + melaza con una rentabilidad de \$2.01 además mostró el menor costo variable unitario \$1.50 teniendo la mejor producción 2.79 $\mathrm{t} \mathrm{ha}^{-1}$. Comparado con el tratamiento de biol + harina de ave + suero + melaza teniendo la menor producción $1.8 \mathrm{t} \mathrm{ha}^{-1}$, la menor rentabilidad $\$ 1.02$, y el mayor costo variable unitario \$2.23, como se muestra en el cuadro 3.

Cuadro 3. Análisis económico de los tratamientos

\begin{tabular}{lcccr}
\hline Tratamientos & $\begin{array}{l}\text { Costo } \\
\text { variable } \\
\text { unitario }\end{array}$ & Ganancia & $\begin{array}{l}\text { Rentabilidad } \\
(\mathbf{B} / \mathbf{C})\end{array}$ & $\begin{array}{l}\text { Producción } \\
\text { T-1 }\end{array}$ \\
\hline Biol, Harina de ave, Suero y & $\$ 2.23$ & $\$ 1.02$ & 1.876 \\
\hline
\end{tabular}

Revista Científica Biológico AgropecuariaTuxpan 2 (1) 


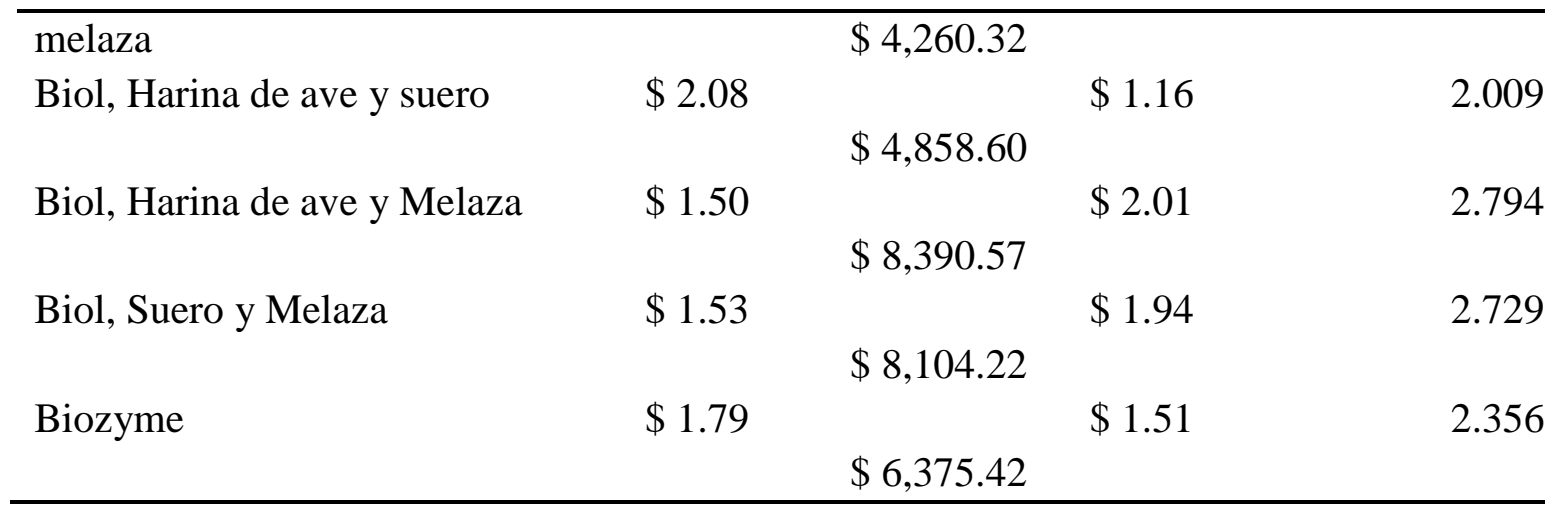

\section{CONCLUSIONES}

Los resultados mostraron que el mejor tratamiento fue las aplicaciones foliares de biol + harina de ave + melaza tienen buena sanidad de mazorca de acuerdo a la escala realizada.

Los menores rendimientos $1.8 \mathrm{t} / \mathrm{h}-1$ y 2 $\mathrm{t} / \mathrm{h}-1$ estuvieron asociados con porcentajes mayor a $20 \%$ de mazorcas dañadas por el hongo F. moniliforme.

El mejor tratamiento que generó el mayor rendimiento de grano de $2.79 \mathrm{t}$ ha-1 fue biol + harina de ave + melaza y por consecuencia obtuvo la mejor rentabilidad que fue de $\$ 2.01$.

Al utilizar el biol se disminuye la contaminación del suelo, aire y mantos freáticos y puede generar buenos resultados en el rendimiento de grano sustituyendo parcialmente la fertilización química.

La aplicación de fertilizantes foliares orgánicos pueden mejorar la economía del pequeño productor e incentivarlos al uso de tecnologías limpias.

\section{LITERATURA CITADA}

Bejarano, C., y H., Méndez. 2004. Fertilización orgánica comparada con la fertilización química en el cultivo de fréjol (Phaseolus vulgaris), para minimizar el efecto de degradación del suelo". Tesis de licenciatura, Facultad de Ingeniería en Ciencias Agropecuarias y Ambientales, Escuela de Ingeniería en Recursos Naturales Renovables, Ibarra Ecuador, $128 \mathrm{pp}$. https://doi.org/10.26820/recimundo/1.5.2017.884-892

Guanopatín-Chicaiza, M. R. 2012. Aplicación de biol en el cultivo establecido de alfalfa (medicago sativa). Tesis Universidad Técnica de Ambato facultad de Ingeniería Agronómica. Cevallos, Ecuador 2012.77 p.

Hernandez-Casillas, J. M., y Esquivel-Esquivel, G. 2004. Rendimiento de grano y características agronómicas en germoplasma de maíz de valles altos de México. Revista Fitotecnia Mexicana. Vol. 27, num. Es1. Sociedad Mexicana de Fitogénetica, A.C. México, pp. 27-31. https://doi.org/10.35196/frm.2012.2.161

\section{Revista Científica Biológico Agropecuaria Tuxpan 2 (1)}


SAGARPA. Proyecto para la Producción de Maíz de Invierno en la Región Sur-Sureste de México (2008-2009). Informe de evaluación de desempeño e indicadores de impacto.

https://doi.org/10.35537/10915/3027

Sierra-Macias, M. E. N., Becerra-Leor, A., Palafox-Caballero, S., Barron-Freyre, O., Cano-Reyes, A., Sambada-Martinez, A., Sandoval-Rincon, J. y Romero-Mora. 2004. Caracterización de híbridos de maíz (Zea mays L.) con alta calidad de proteína por su rendimiento y tolerancia a pudrición de mazorcas en el sureste de México. Revista Mexicana de Fitopatología. Vol 22, numero 002. Sociedad Mexicana de Fitopatología, A.C. México, pp. 268-276.

https://doi.org/10.15517/am.v18i1.5040
Tinoco, A. C. A., Rodríguez, M. F. A., Sandoval, R. J. A., Barrón, F. S., Palafox, C. A., Esqueda, E. V. A., Sierra, M. M., Romero, M. J. 2002. Manual de producción de maíz para los estados de Veracruz y Tabasco. INIFAP. CIRGOC. Campo Experimental Papaloapan. Libro Técnico Núm. 9. Veracruz, México. 113 pp.

Tinoco-Alfaro, C. A. A., Ramírez-Fonseca, E., Villareal-Farías, A. y Ruiz-Corral. 2008. Arreglo espacial de híbridos de maíz, índice de área foliar y rendimiento. Instituto Nacional de Investigaciones Forestales Agrícolas y Pecuarias. Agricultura Técnica en México Vol. 34 Núm. 3, p. 271-278.

https://doi.org/10.24850/j-tyca-2017-03-04

Copyright (c) 2014Mario Alejandro Hemández Chontal, Ariadna Linares Gabriel, Carlos Alberto Tinoco Alfaro y Nereida Rodriguez Orozco

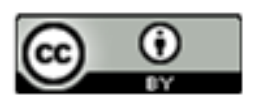

Este texto está protegido por una licencia Creative Commorr 4.0 .

Usted es libre para Compartir —copiar y redistribuir el material en cualquier medio o formato- y Ad aptar el documento —remezclar, transformar y crear a partir del material- para cualquier propósito, inchso para fines comerciales, siempre que cumpla la condición de:

Atribución Usted debe dar crédito a la obra original de manera adecuada, proporcionar un enlace a la licencia, e indicar si se han realizado cambios. Puede hacerlo en cualquier forma razonable, pero no de forma tal que sugiera que tiene el apoyo del licenciante o lo recibe por el usoque hace de la obra.

Resumende licencia - Textocompletodelalicencia 